\title{
Analytical Binary Modeling and its Role in Dynamics
}

\author{
Tapan K. Chatterjee \\ Department of Physics, University of the Americas, Puebla, Mexico \\ e-mail chtapan@yahoo.com
}

\section{Introduction and Theory}

Binaries are critical tools that drive stellar systems; which play a major role in galactic dynamics. Internal evolution of globular clusters, e.g., due to evolution of binaries, can be modulated by external effects, like tidal shocks (e.g., Chernoff \& Weinberg 1990). There are well-established relations between dynamical properties of globular clusters and their galactrocentric distance, $\mathrm{R}(\mathrm{g})$. A characteristic one is: $\mathrm{D}(1 / 2) \approx[\mathrm{R}(\mathrm{g})]^{1 / 2}$, where $\mathrm{D}(1 / 2)$ is the diameter containing half the mass / luminosity in projection (van den Bergh et al. 1991). This relation could be a result of tidal evaporation of clusters and feedback of forming protoclusters (Surdin, 1995). A similar limitation of globular cluster distributions is obtained in the half-mass density - kinetic temperature plot and used in many cluster formation scenarios (Muray \& Lin 1992). This relationship should have an explanation on the basis of dynamic evolution of the cluster population.

This merits a study of orbital characteristics of binary formation and its applications. In Magalinsky \& Chatterjee (2000), we formulate the Hamiltonian treatment of the dynamics of two extended bodies, corresponding to their separation and effective sizes. The internal movement of the constituents of the bodies is taken into account by the hydrodynamic velocities corresponding to their mass distribution and the gravitational potential is considered in the form of a flux. A study of binary motion under spinorbit synchronization and a condition which favors energy minimization is conducted. Considering binaries to be subsystems in a microcanonical ensemble, we find that for bound binary systems the equipartition distribution of the system parameters favors high eccentricities.

\section{Results and Conclusions}

Post-collapse evolution of globular clusters is driven by energy output of hard binaries. This model is of such (hard) type and indicates that most of these eccentric binaries will harden by absorbing energy and lowering their eccentricities (e.g., Hut et al. 1992, for a review). Numerical simulations (Chatterjee \& Maganinsky 2002) and observations (Chengalur et al. 1996 and references therein) indicate that high eccentricities are maintained for a large timescale; circularization takes place in the adiabatic regimen (Chatterjee \& Magaslinsky 2006), but on a very large time-scale. Eccentric binaries enhance collisions, as the cross-sections for close encounters depend upon eccentricity: The probability is about an order of magnitude more for highly eccentric binaries as compared to nearcircular ones. In three-body encounters the binary is hardened and the single star gains velocity. Thus the core of the cluster is gradually heated; this is a major source of energy input that drives the post-collapse evolution (e.g., Goodman \& Hut 1993). Preliminary results indicate that this model of hard binaries is likely to stabilize post-collapse oscillations (Chatterjee \& Magalinsky 2006). 
The model also throws light on the aspects of dynamical evolution of globular clusters that lead to the correlation between half-mass density and kinetic temperature, in the sense that in-three body encounters the core is gradually heated, and eccentric binaries enhance this collision process (Chatterjee \& Magalinsky 2006).

\section{References}

Chatterjee, T.K. \& Magalinsky, V.B. 2002, Astron. Astrophys. Trans. 21, 79

Chatterjee, T.K. \& Magalinsky, V.B. 2006, Astron. Astrophys. Tran. in press

Chengalur, J.N., Salpeter, E.E., \& Terzian, Y. 1996, ApJ, 461, 546

Chernoff, D. \& Weinberg, M. 1990, ApJ 351, 121

Goodman, J. \& Hut, P. 1993, ApJS 85, 347

Hut, P. et al. 1992, PASP 105, 981

Magalinsky, V.B. \& Chatterjee, T.K. 2000, Astron. Astrophys. Trans. 18, 807

Muray, S.D. \& Lin, D.N.C. 1992, ApJ 400, 265

Surdin, V.G. 1995, Astron. Let. 21, N4

Van den Bergh, S., Morbey, C., \& Pazder, J. 1991, ApJ 375, 594. 